important results from this study is the identification of ADRB downstream signaling as a potential predictive factor for patients likely to gain benefit from adjuvant beta blocker therapy while receiving androgen ablation therapy.

\section{Conclusions}

Although the current study provides a new mechanistic understanding of the effects of stress on cancer progression, there remain many unanswered questions with regard to such effects. Among these is the identification of specific patient subsets that are most likely to benefit from interventions targeted against stress-related pathways. Whether such subsets should be identified based on behavioral and/or molecular features is currently unknown. Moreover, identifying reliable downstream markers to test the efficacy of stress-based interventions may allow a more rational selection of therapies. Much like cancer, stress pathways are extremely complex, and it is unclear whether SNS-targeted interventions will be sufficient or whether blocking other pathways, such as HPA mediators or inflammation, will also be required. Addressing these and other questions will be an important component of realizing the full translational potential of the preclinical findings presented in this and other research. Nevertheless, the field continues to rapidly evolve, and novel signaling mechanisms are being discovered that provide a deeper understanding of the effects of behavioral stress on tumor biology. The present study moves the field forward by demonstrating that behavioral stress enables prostate cancer cells to evade apoptosis, an important characteristic in the process of tumor growth and metastasis. In addition, the authors provide a new understanding of mechanisms by which prostate cancer cells could acquire resistance to androgen therapy.

\section{Acknowledgments}

The authors are supported by NIH grants (CA140933, CA104825, CA109298, P50CA083639, P50CA098258, CA128797, U54CA151668), an Ovarian Cancer Research Fund Program Project Development Grant, the Department of Defense (OC073399, W81XWH-10-1-0158, OC100237), the Betty Ann Asche Murray Distinguished Professorship, the Marcus Foundation, and the Blanton-Davis Ovarian Cancer Research Program.

Address correspondence to: Anil K. Sood, Departments of Gynecologic Oncology and Cancer Biology, The University of Texas MD Anderson Cancer Center, Unit 1362, PO Box 301439, Houston, Texas 77230-1439, USA. Phone: 713.745.5266; Fax: 713.792.7586; E-mail: asood@mdanderson.org.

1. Antoni MH, et al. The influence of bio-behavioural factors on tumour biology: pathways and mechanisms. Nat Rev Cancer. 2006;6(3):240-248.

2. Thaker $\mathrm{PH}$, et al. Chronic stress promotes tumor growth and angiogenesis in a mouse model of ovar- ian carcinoma. Nat Med. 2006;12(8):939-944.

3 . Sood AK, et al. Adrenergic modulation of focal adhesion kinase protects human ovarian cancer cells from anoikis. J Clin Invest. 2010;120(5):1515-1523.

4. Sood AK, et al. Stress hormone-mediated invasion of ovarian cancer cells. Clin Cancer Res. 2006;12(2):369-375.

5. Yang EV, et al. Norepinephrine upregulates VEGF, IL-8, and IL-6 expression in human melanoma tumor cell lines: implications for stress-related enhancement of tumor progression. Brain Behav Immun. 2009;23(2):267-275.

6. Armaiz-Pena GN, Cole SW, Lutgendorf SK, Sood AK. Neuroendocrine influences on cancer progression [published online ahead of print June 21, 2012]. Brain Behav Immun. doi:10.1016/j. bbi.2012.06.005.

7. Lutgendorf SK, et al. Social influences on clinical outcomes of patients with ovarian cancer. J Clin Oncol. 2012;30(23):2885-2890.

8. Volden PA, Conzen SD. The influence of glucocorticoid signaling on tumor progression [published online ahead of print November 16, 2012]. Brain Behav Immun. doi:10.1016/j.bbi.2012.10.022.

9. Hassan S, et al. Behavioral stress accelerates prostate cancer development in mice. J Clin Invest. 2013;123(2):874-886

10. Hanahan D, Weinberg RA. The hallmarks of cancer. Cell. 2000;100(1):57-70.

11. McKenzie S, Kyprianou N. Apoptosis evasion: the role of survival pathways in prostate cancer progression and therapeutic resistance. J Cell Biochem. 2006;97(1):18-32

12. Taichman RS, Loberg RD, Mehra R, Pienta KJ. The evolving biology and treatment of prostate cancer. J Clin Invest. 2007;117(9):2351-2361.

13. Feldman BJ, Feldman D. The development of androgen-independent prostate cancer. Nat Rev Cancer. 2001;1(1):34-45.

14. Sastry KS, et al. Epinephrine protects cancer cells from apoptosis via activation of cAMP-dependent protein kinase and BAD phosphorylation. J Biol Chem. 2007;282(19):14094-14100.

15. Lutgendorf SK, et al. Social isolation is associated with elevated tumor norepinephrine in ovarian carcinoma patients. Brain Behav Immun. 2011;25(2):250-255.

\title{
iRHOM2 takes control of rheumatoid arthritis
}

\author{
Stefan F. Lichtenthaler
}

German Center for Neurodegenerative Diseases (DZNE), Munich, Germany. Technische Universität München, Munich, Germany. Munich Cluster for Systems Neurology (SyNergy), Munich, Germany.

\begin{abstract}
The cytokine TNF- $\alpha$ is a major drug target for rheumatoid arthritis, an inflammatory joint disorder. An alternative approach is to target the protease TNF- $\alpha$ convertase (TACE), which releases TNF- $\alpha$ from cells. However, because TACE cleaves other proteins involved in development and cancer, a tissue-specific inhibition of TACE in immune cells appears mandatory. In this issue of the JCI, Issuree et al. report that iRHOM2 is a TACE activator in immune cells. Loss of iRHOM2 largely protects mice from inflammatory arthritis, making iRHOM2 a potential drug target for this condition.
\end{abstract}

Conflict of interest: The author has declared that no conflict of interest exists.

Citation for this article: $J$ Clin Invest. 2013; 123(2):560-562. doi:10.1172/JCI67548.
iPad, iPod, iPhone - iRHOM sounds like the latest gadget you must have. There are even two iRHOM versions. iRHOM1 (also known as RHBDF1) appears to have broad functionality, whereas iRHOM2 (also known as RHBDF2) has more restricted and exclusive functions. iRHOMs are proteolytically inactive homologs of rhomboid proteases. They localize to the membrane of the ER and were initially shown to be part of the ER protein quality control machinery both in Drosophila and mammalian cells (1). Three recent studies demonstrated that an additional function, at least for iRHOM2, is mediating the release of TNF- $\alpha$ from macrophages (2-4). iRHOM2 acts as a cargo receptor 
A

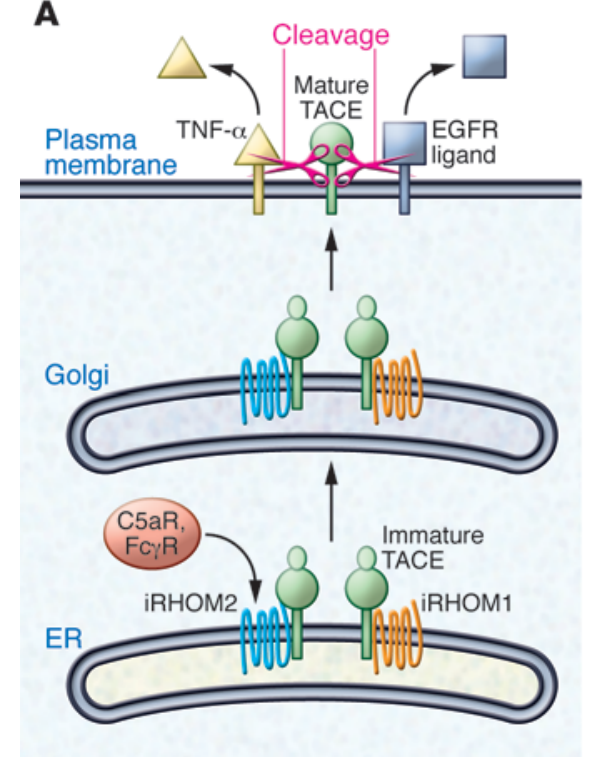

B

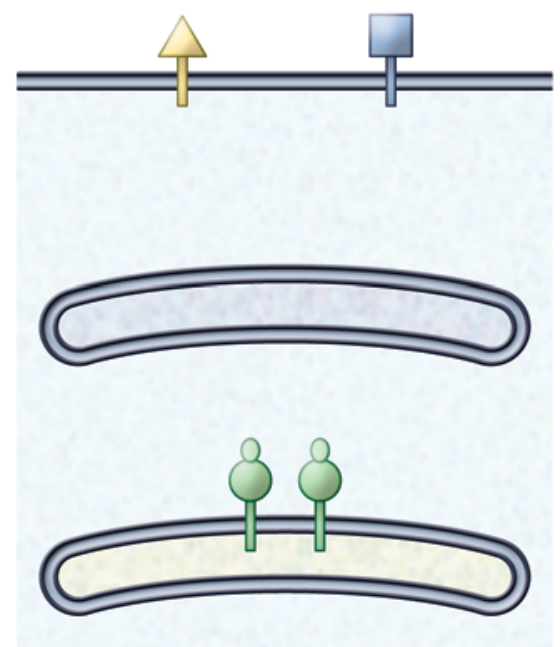

C

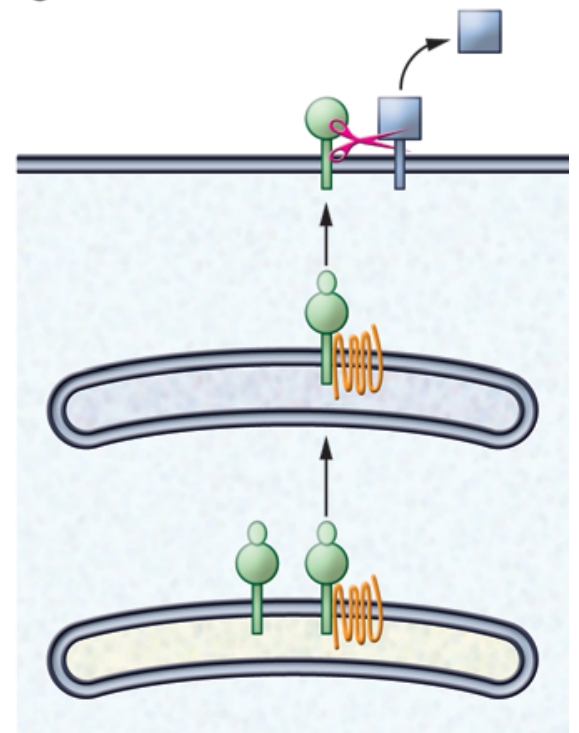

Figure 1

Control of TACE maturation by iRHOM1 and iRHOM2. (A) In wild-type cells, iRHOM1 or iRHOM2 associate with immature TACE and promote TACE exit from the ER. In the Golgi, TACE undergoes maturation and activation and travels without the iRHOMs to the plasma membrane (PM), where it cleaves TNF- $\alpha$ and other TACE ligands, such as ligands for the EGFR. C5a receptor (C5aR) and Fc $\gamma$ receptor (FcyR) activate iRHOM2 expression and TACE maturation in the immune system. (B) Immune cells lack significant iRHOM1 expression. Upon knockout of iRHOM2, TACE can no longer mature in immune cells. As a result, TNF- $\alpha$ is not cleaved, providing protection from TNF- $\alpha-$ dependent diseases, such as RA and sepsis. (C) iRHOM1 is expressed in nonimmune cells. In the absence of iRHOM2, iRHOM1 is sufficient for TACE activation, allowing TACE substrates to be cleaved normally in nonimmune cells.

that binds TNF- $\alpha$ convertase (TACE) (also known as ADAM17) in the ER and helps TACE to get to the plasma membrane (Figure 1A). There, TACE cleaves the membrane-bound TNF- $\alpha$ precursor, resulting in release of the soluble TNF- $\alpha$ cytokine. Consequently, genetic inactivation of iRHOM2 blocks TACE activity at the cell surface of macrophages and other immune cells, largely preventing TNF- $\alpha$ release (Figure 1B). TNF- $\alpha$ acts as key pathologic player in inflammatory diseases, such as rheumatoid arthritis (RA), sepsis, and psoriasis, but is also required for innate immunity. An impairment of TNF- $\alpha$ production has been described in iRHOM2-deficient mice, making them resistant to LPS-induced septic shock, although they succumb more rapidly to bacterial infection $(2,3)$.

In this issue of the JCI, Issuree et al. assessed the involvement of iRHOM2 in another TNF- $\alpha$-dependent disease, RA, using the $\mathrm{K} / \mathrm{BxN}$ mouse model (5). This model mimics human RA (6), even though it is not fully dependent on the production of TNF- $\alpha$. Serum transfer from the K/BxN mouse induced joint swelling and cartilage erosion in wild-type mice, but this effect was ameliorated with iRHOM2 deficiency. Likewise, the clinical scores, which indicate the extent of the tissue damage, were greatly improved in the absence of iRHOM2 (5). Similar results were obtained in mice lacking TACE specifically in myeloid cells and, as previously reported, in TNF- $\alpha$-deficient mice (7). These data convincingly show that iRHOM2-deficient mice are largely protected from inflammatory arthritis because of the myeloid cell-specific block of TACE activity and the resulting prevention of TNF- $\alpha$ release (Figure 1B). Given the role of iRHOM2 in sepsis (3) and RA, it is likely that iRHOM2 also contributes to the pathogenesis of other TNF- $\alpha$-dependent diseases, such as psoriasis.

\section{Propagating the signal}

Inflammatory arthritis can be induced in mice by the transfer of serum from $\mathrm{K} / \mathrm{BxN}$ mice, but this requires that the recipients express complement C5a receptor and Fcy receptor (8). However, the molecular mechanisms linking receptor activation and TNF- $\alpha$ production are not understood in detail. Issuree et al. now demonstrate a role for iRHOM2 and TACE in this process (5). In murine macrophages or human monocytes, the activation of C5a receptor and Fc $\gamma$ receptor increased TNF- $\alpha$ production, which was blocked by iRHOM2 knockdown or TACE inhibition, clearly implicating both proteins in the increased TNF- $\alpha$ production. Receptor activation strongly increased iRHOM2 expression, which is a mechanism to activate TACE and increase TNF- $\alpha$ release (3). Surprisingly, however, not only mature, but also immature, TACE levels were increased. This indicates that receptor activation also regulates TACE expression levels, which may be an additional mechanism besides increased iRHOM2 expression to increase TNF- $\alpha$ release. Whether these mechanisms are additive or redundant for TNF- $\alpha$ production remains to be tested.

\section{Location, location, location}

TACE is ubiquitously expressed and has major substrates and functions besides TNF- $\alpha$ cleavage $(9,10)$. In fact, mice with global TACE deletion die perinatally as a result of reduced EGFR signaling during development (11), and study of a TACE-deficient patient demonstrated essential roles for TACE in skin and intestine (12). Thus, it is surprising that iRHOM2-deficient mice show a myeloid cell-specific - and not a general - block of TACE activity. The explanation comes from the expression profiles of iRHOM2 and its homolog iRHOM1 and the new finding that iRHOM1 can have a 
similar function as iRHOM2 in promoting TACE activity and TACE substrate cleavage (5). In other words, iRHOM1 can functionally compensate for the loss of iRHOM2 and vice versa, at least in tissues in which both proteins are expressed. As shown by Issuree et al. in this issue of the JCI (5) and by others previously $(2,3)$, iRHOM2 expression is high in myeloid cells, in which iRHOM1 is expressed at low levels and cannot functionally compensate for iRHOM2, leading to the observed loss of TACE function in iRHOM2-deficient macrophages as well as in lymph nodes and bone marrow (Figure $1 \mathrm{~B}$ ). In contrast, in mouse embryonic fibroblasts, in which both iRHOM1 and iRHOM2 are expressed, the single loss of either iRHOM1 or iRHOM2 did not affect TACE maturation (Figure 1C), which is a frequently used readout for TACE activity. However, loss of both proteins prevented TACE maturation, demonstrating functional redundancy of iRHOM1 and iRHOM2 in mouse embryonic fibroblasts. Additionally, several organs from iRHOM2-deficient mice were analyzed for TACE maturation. No major changes were observed, suggesting sufficient iRHOM1 compensation in these organs. Whether mild changes in TACE maturation or even TACE levels observed in some organs correlate with reduced iRHOM1 expression remains to be resolved in future studies.

\section{Conclusions}

Taken together, the new study by Issuree et al. and other recent work suggest that inactivation of iRHOM2 - even if it happens in the whole body - is an elegant way to obtain a tissue-specific TACE inactivation in immune cells while maintaining critical TACE functions in other organs. This makes iRHOM2 an attractive drug target for TNF- $\alpha$-dependent diseases, such as RA and sepsis. Whether iRHOM2 can be a safe drug target, in particular upon chronic inhibition, remains to be seen. For example, in multiple sclerosis, it remains unclear whether TNF- $\alpha$ reduction is beneficial or harmful $(13,14)$. Additionally, iRHOM2deficient mice succumb more readily to bacterial infections (3), a clear side effect of the reduced TNF- $\alpha$ production. Similarly, patients treated with current TNF- $\alpha$ blockers appear to be at increased risk for serious infections, such as tuberculosis (15). Nevertheless, these TNF- $\alpha$ blockers impressively demonstrate that TNF- $\alpha$ reduction is therapeutically successful for different TNF- $\alpha-$ dependent inflammatory diseases, and this may also be true for iRHOM2 inhibition. It needs to be considered, though, that point mutations in iRHOM2 were recently genetically linked to tylosis, a disease that causes palmoplantar keratoderma and a high risk of oesophageal cancers (16). It is not yet known whether the mutations lead to a gain or loss of function of iRHOM2, but they appear to increase EGFR signaling through as yet unknown molecular mechanisms. The mutations may affect TACE, which cleaves EGFR ligands; they could block the previously reported function of iRHOM2 in ER-associated degradation of EGFR ligands (1); or they could affect as yet unknown iRHOM2 client proteins other than TACE or EGFR ligands. The above considerations demonstrate that a critical evaluation of the therapeutic potential of iRHOM2 requires a more comprehensive understanding of its (patho)physiological functions, its client spectrum, and its potential cross-talk with iRHOM1.

Is iRHOM2 a druggable target? At a first glance, the answer may be no, because the membrane protein iRHOM2 is not an enzyme, and it is largely buried in the ER membrane. However, this is also true for presenilin 1 and 2, which are the catalytic subunits of the protease $\gamma$-secretase and are major targets in the development of Alzheimer's disease therapeutics; small-molecule presenilin inhibitors have been developed successfully. Some of them even appear to preferentially block presenilin 1 over its homolog presenilin 2 (17). Likewise, specificity to iRHOM2 over iRHOM1 would be needed for the TNF- $\alpha$-dependent diseases.

In summary, the new study in this issue by Issuree et al. provides three major advances in our understanding of the biology and medical importance of iRHOMs (5). First, this work demonstrates that iRHOM2 is relevant for another TNF- $\alpha-$ dependent disease, RA. Second, the study provides insights into the regulation of iRHOM2 expression and the molecular mechanisms of RA. Third, it provides evidence that the homolog iRHOM1 has a similar function as iRHOM2 in controlling TACE activity. This is relevant for evaluating iRHOM2 as a potential drug target in RA and other TACE-dependent diseases. The new discoveries about iRHOM2, but also the many remaining open questions, show that this is an exciting time for iRHOM research. The new work by Issuree et al. brings us back to the initial question: are iRHOM1 and iRHOM2 gadgets that you must have? The data presented here suggest that, as is often the case with iGadgets, selection of the appropriate version may be personal and context dependant.

\section{Acknowledgments}

I thank the BMBF (KNDD) and the Friedrich Baur Foundation for financial support.

Address correspondence to: Stefan F. Lichtenthaler, German Center for Neurodegenerative Diseases (DZNE), Max-LebschePlatz 30, 81377 Munich, Germany. Phone: 49.89.7095.8416; Fax: 49.89.7095.8420; E-mail: stefan.lichtenthaler@dzne.de.

1. Zettl M, Adrain C, Strisovsky K, Lastun V, Freeman M. Rhomboid family pseudoproteases use the ER quality control machinery to regulate intercellular signaling. Cell. 2011;145(1):79-91.

2. Adrain C, Zettl M, Christova Y, Taylor N, Freeman $M$. Tumor necrosis factor signaling requires iRhom 2 to promote trafficking and activation of TACE. Science. 2012;335(6065):225-228.

3. McIlwain DR, et al. iRhom2 regulation of TACE controls TNF-mediated protection against Listeria and responses to LPS. Science. 2012;335(6065):229-232.

4. Siggs OM, et al. iRhom 2 is required for the secretion of mouse TNFalpha. Blood. 2012; 119(24):5769-5771.

5. Issuree PDA, et al. iRHOM2 is a critical pathogenic mediator of inflammatory arthritis. J Clin Invest. 2013;123(2):928-932.

6. Ditzel HJ. The $\mathrm{K} / \mathrm{BxN}$ mouse: a model of human inflammatory arthritis. Trends Mol Med. 2004;10(1):40-45.

7. Ji H, et al. Critical roles for interleukin 1 and tumor necrosis factor alpha in antibody-induced arthritis. J Exp Med. 2002;196(1):77-85.

8. Ji H, et al. Arthritis critically dependent on innate immune system players. Immunity. 2002; 16(2):157-168.

9. Scheller J, Chalaris A, Garbers C, Rose-John S. ADAM17: a molecular switch to control inflammation and tissue regeneration. Trends Immunol. 2011;32(8):380-387.

10. Lichtenthaler SF, Haass C, Steiner H. Regulated intramembrane proteolysis - lessons from amyloid precursor protein processing. J Neurochem. 2011;117(5):779-796

11. Peschon JJ, et al. An essential role for ectodomain shedding in mammalian development. Science. 1998;282(5392):1281-1284.

12. Blaydon DC, et al. Inflammatory skin and bowel disease linked to ADAM17 deletion. $N$ Engl J Med. 2011;365(16):1502-1508.

13. Brambilla R, et al. Inhibition of soluble tumour necrosis factor is therapeutic in experimental autoimmune encephalomyelitis and promotes axon preservation and remyelination. Brain. 2011; 134(pt 9):2736-2754.

14. Gregory AP, et al. TNF receptor 1 genetic risk mirrors outcome of anti-TNF therapy in multiple sclerosis. Nature. 2012;488(7412):508-511.

15. Moss ML, Sklair-Tavron L, Nudelman R. Drug insight: tumor necrosis factor-converting enzyme as a pharmaceutical target for rheumatoid arthritis. Nat Clin Pract Rheumatol. 2008;4(6):300-309.

16. Blaydon DC, et al. RHBDF2 mutations are associated with tylosis, a familial esophageal cancer syndrome. Am J Hum Genet. 2012;90(2):340-346.

17. Borgegard T, et al. Alzheimer's disease: presenilin 2-sparing gamma-secretase inhibition is a tolerable Ab peptide-lowering strategy. J Neurosci. 2012;32(48):17297-17305. 